OPEN ACCESS

Edited by:

Christopher J. Hasson,

Northeastern University, USA

Reviewed by:

Claudia Gianelli,

University of Potsdam, Germany

Antonella Maselli,

Fondazione Santa Lucia (IRCCS), Italy Bastien Berret,

University Paris-Sud - Université

Paris-Saclay, France

*Correspondence:

Cristina Becchio

cristina.becchio@unito.it

Received: 16 March 2016 Accepted: 06 September 2016 Published: 22 September 2016

Citation:

Ansuini C, Cavallo A, Campus C, Quarona D, Koul A and Becchio C (2016) Are We Real When We Fake?

Attunement to Object Weight in Natural and Pantomimed Grasping

Movements.

Front. Hum. Neurosci. 10:471. doi: 10.3389/fnhum.2016.00471

\section{Are We Real When We Fake? Attunement to Object Weight in Natural and Pantomimed Grasping Movements}

\author{
Caterina Ansuini ${ }^{1}$, Andrea Cavallo ${ }^{2}$, Claudio Campus ${ }^{3}$, Davide Quarona ${ }^{1}$, Atesh Koul ${ }^{1}$ \\ and Cristina Becchio ${ }^{1,2 *}$ \\ ${ }^{1}$ C'MON Unit, Fondazione Istituto Italiano di Tecnologia, Genova, Italy, ${ }^{2}$ Department of Psychology, University of Turin, \\ Torino, Italy, ${ }^{3}$ U-VIP Unit, Fondazione Istituto Italiano di Tecnologia, Genova, Italy
}

Behavioral and neuropsychological studies suggest that real actions and pantomimed actions tap, at least in part, different neural systems. Inspired by studies showing weightattunement in real grasps, here we asked whether (and to what extent) kinematics of pantomimed reach-to-grasp movement can reveal the weight of the pretended target. To address this question, we instructed participants $(n=15)$ either to grasp or pretend to grasp toward two differently weighted objects, i.e., a light object and heavy object. Using linear discriminant analysis, we then proceeded to classify the weight of the target - either real or pretended - on the basis of the recorded movement patterns. Classification analysis revealed that pantomimed reach-to-grasp movements retained information about object weight, although to a lesser extent than real grasp movements. These results are discussed in relation to the mechanisms underlying the control of real and pantomimed grasping movements.

Keywords: reach-to-grasp, pantomime, object weight, kinematics, linear discriminant analysis

\section{INTRODUCTION}

The ability to reach out and grasp objects with considerable skill is one of the defining features of primates. In both humans and non-human primates, prehension is typically directed at a visible object and results in contact with the object, manipulation, and haptic feedback. Humans (and perhaps some other species, Douglas and Moscovice, 2015), however, are also capable of grasping an imaginary object. The interest in this ability, both for clinical and theoretical investigation, is due to its double nature (Goldenberg, 2013). Pantomimed actions are derived from instrumental actions of actual use. They are, however, communicative gestures in that they involve the repetitions of instrumental movements, but without acting on an object, as a way of communicating something about the action or the object.

This double nature of pantomime - both instrumental and communicative at the same time is reflected in the differential use that real and pantomimed grasps make of object knowledge. In real grasps, knowledge about objects and their manipulation is used to conform the hand gradually to the properties of the object to be grasped. For example, when grasping a glass, scaling of grip width to the width of the glass is achieved by first opening the hand in proportion to, but wider than the visually perceived width of the glass, and then closing it around the glass, ensuring a safety 
margin for grasping the object securely (Jeannerod, 1988; but see also Smeets and Brenner, 1999 for an alternative account of this effect).

In contrast, in pantomimed grasps, knowledge about objects is converted into actions that demonstrate the perceptual distinctive features of the pretended objects (Goldenberg et al., 2007). This conversion necessitates the selection of some features of the actual grasp, while permitting one to neglect others, i.e., those features that adapt the hand to the material object. Thus, when pantomiming, for instance, participants do not show grip 'overshoot', but open the hand to the approximate width of the pretended object 'to depict' its width (Goodale et al., 1994). One particular difficulty of pantomimed grasp tasks relates, therefore, to the transformation of object features into a non-routine movement sequence that demonstrates the perceptual features of the pretended object (Goldenberg et al., 2003).

Although previous research indicates that pantomimed grasp incorporates spatial features of a pretended target, such as its actual (Goodale et al., 1994; Cavina-Pratesi et al., 2011) or visually perceived size (Westwood et al., 2000), it is questionable whether pantomimed grasp can also demonstrate non-spatial features of the target. Specification of object size requires selecting a simple spatial characteristic of the object (e.g., the width of the object) and converting it into a spatial relationship between a limited set of discrete body parts (e.g., the distance between thumb and index). Arguably, depicting a non-spatial characteristic of the object, such as its weight or fragility, might be more complicated as no simple perceptual matching is possible for transforming the representation of the weight or the fragility of an object into a distinctive grasping pattern.

Here we set out to examine the representational reach of pantomime by asking whether pantomimed grasping can transmit information about the weight of a pretended object.

\section{Influence of Object Weight on Action Planning and Control}

Object weight has been shown to influence visuo-motor planning and control of real grasps (Weir et al., 1991; Brouwer et al., 2006; Eastough and Edwards, 2007). For example, Eastough and Edwards (2007) observed that heavy compared to light objects caused greater peak grip aperture and the opposing placement of the index finger and thumb. This effect of weight on grasping kinematics has been proposed to directly reflect the requirements for a stable grasp (see Smeets and Brenner, 1999 for a review). When grasping heavy objects, to reduce the chances of object rotation and slippage, fingers should be positioned accurately enough so that the grip position passes through the center of mass of the object to be grasped. It is perhaps not surprising, therefore, that weight influences pre-contact kinematics of real grasp movements.

In contrast to real grasps, however, pantomimed grasps entail no preparation for a stable final grip placement on the object. After all, the pretended target is 'weightless' and there is no risk of slippage or rotation. The influence of object weight on pantomimed grasps, if any, would thus reflect the pure effort to 'depict' the weight of the imagined object by translating a nonspatial property of the object into distinctive features of a motor act.

To determine whether (and to what extent) kinematics of a pantomimed grasp can reveal the weight of the pretended target, in the present study, we first recorded the kinematics of real grasping and pantomimed grasping movements toward differently weighted objects. Using linear discriminant analysis (LDA), we then proceeded to classify the weight of the target either real or pretended - on the basis of the recorded movement patterns. This innovative approach combining kinematics with classification methods allowed us to obtain a measure of weightrelated information transmitted by the hand movements over time.

\section{MATERIALS AND METHODS}

\section{Participants}

Fifteen participants took part in the study. They had a mean age of 26.8 years (SD: 2.2; range: $24-32$ years old; 5 males) and were all right handed, with normal or corrected-to-normal vision, and with no history of either psychiatric or neurological disorders. The experimental procedures were approved by the local ethical committee (ASL 3 Genovese) and were carried out in accordance with the principles of the revised Helsinki Declaration (World Medical Association General Assembly, 2008). Each participant provided written informed consent and was paid in return for participation.

\section{Apparatus and Procedures}

Participants were seated on a height-adjustable chair with the right elbow and wrist resting on a table, the forearm pronated, the arm oriented in the parasagittal plane passing through the shoulder, and the right hand in a semi-pronated position, with the tips of the thumb and index finger placed, in gentle opposition, on a tape-marked point. This posture as well as the angular orientation of the wrist were controlled so as to guarantee the consistency of the start position across participants. The working space was set on the surface of a table (width $=140 \mathrm{~cm}$; length $=70 \mathrm{~cm}$; see Figure 1A) covered with a black cloth. A glass (height $=11 \mathrm{~cm}$; diameter $=8 \mathrm{~cm}$ ) was presented on each trial. Depending on the condition, the glass could be empty (i.e., light object; weight $=139 \mathrm{~g}$; see Figure 1B) or filled with iron screws (i.e., heavy object; weight $=838$ g; see Figure 1B).

In the 'real grasp' task, participants were requested to reach toward, grasp, pick up either the empty or filled glass, and place it on a platform (height $=7 \mathrm{~cm}$; width $=9 \mathrm{~cm}$; length $=9 \mathrm{~cm}$ ), located to the left of the target; see Figure 1A). The glass was positioned at a distance of about $48 \mathrm{~cm}$ from the participant's body midline with which it was aligned. The angle between the sagittal plane passing through the object and the hand start position was equal to about $35^{\circ}$ (see Figure 1A).

In the 'pantomimed grasp' task, the glass, either empty or filled, was positioned at a displaced location (see Figure 1A; dashed line circle). Participants were instructed to imagine that an identical glass was positioned at the target position and were 
A

\section{I}

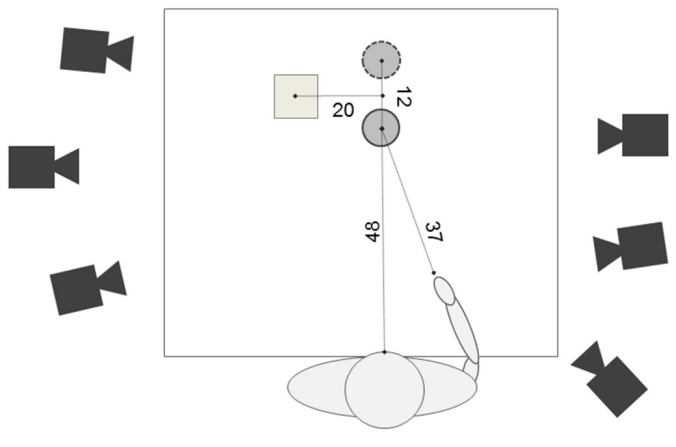

B

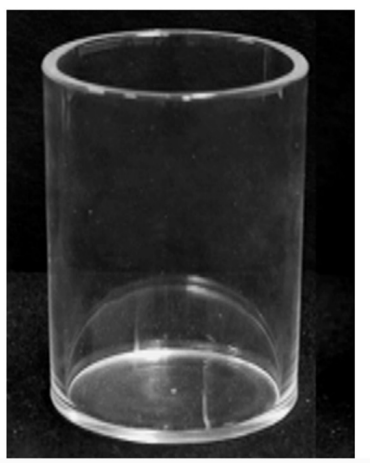

$\checkmark$

\section{C}
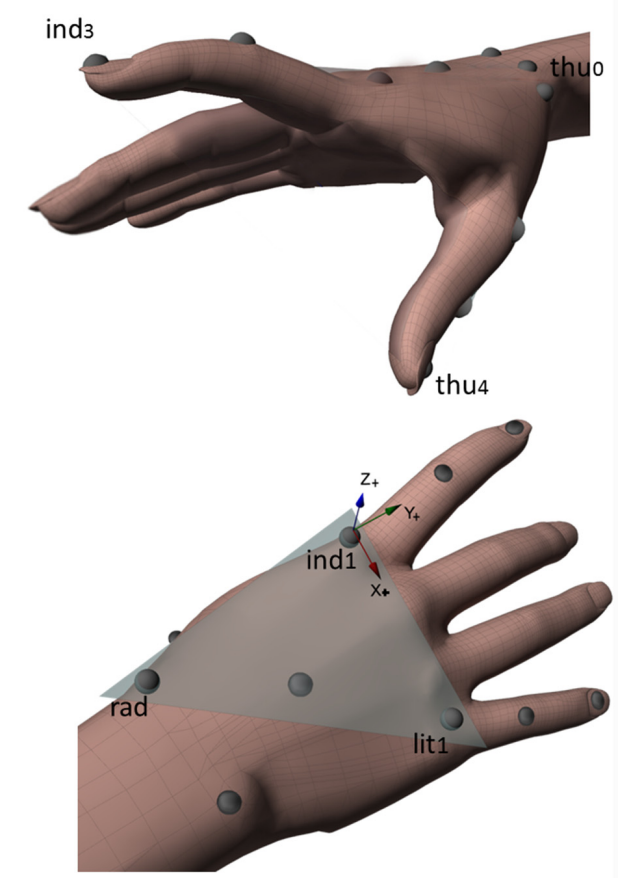

FIGURE 1 | Experimental set-up and hand models for kinematics parameters computation. (A) A schematic representation of the top view of the experimental set-up (not to scale). The position of the object in real grasp task and in pantomimed grasp task is indicated with a filled and a dashed line circle, respectively. Distances are provided in centimeters. (B) A picture of light and heavy object used as target objects. (C) The hand model used to compute kinematics parameters together with a graphical representation of the local frame of reference (Flocal). Flocal had its origin in the marker placed at the metacarpo-phalangeal joint of the index (see ind1). Vectors (ind 1 - lit1) and (ind1 - rad) defined the metacarpal plane of the hand (shaded triangle). In this frame of reference, the $x$-axis had the direction of the vector (ind 1 - lit1; refer to the red arrow) and pointed ulnarly, the $z$-axis was normal to the metacarpal-phalangeal plane, pointing dorsally (refer to the blue arrow), while the $y$-axis was calculated as the cross-product of $z$ - and $x$-axes, pointing distally (refer to the green arrow).

asked to pretend to perform the very same action sequence toward the imagined glass (for a similar paradigm see Goodale et al., 1994).

In both real and pantomimed grasp tasks, participants started the reach-to-grasp movement after a verbal signal from the experimenter. They were instructed to return to the start position and resume hand posture once they were finished placing the glass (or the pretended glass) over the platform. Then, the experimenter returned the glass (if any) to the target position. To ensure that the position of the target object did not vary from trial to trial, for both tasks the glass was placed in between two short pegs that were fixed at the table, the distance between the center of the glass in the real and the pantomimed grasp task being equal to $12 \mathrm{~cm}$ (see Figure 1A).

In each experimental session, a total of 96 trials were administered in eight separate blocks of 12 trials, i.e., two for each type of movement by object weight combination. Blocks were presented in a fixed order. For each object weight, participants performed the real grasp task followed by the pantomimed grasp task. This was done to allow actual experience with object weight and to prevent spurious weight crossover effects when transitioning from the real grasp to the pantomimed grasp task. The order of presentation of object weight was counterbalanced across participants. On average, the time between trials was $15 \mathrm{~s}$ and that between the blocks was $90 \mathrm{~s}$.

At the beginning of each block, the position of the glass (either target or displaced) signaled participants the type of action to be performed (real vs. pantomimed grasp, respectively). Before the experimental session, participants completed 12 practice trials (in four blocks of three trials for each object weight and type of action combination). Block order within the practice session was the same as that adopted during the experimental session. A 2 min pause was allowed between the practice and experimental session. The entire experiment lasted about $60 \mathrm{~min}$.

\section{Movement Recordings and Kinematics Parameters}

To track the kinematics of the hand, we used a nearinfrared camera motion capture system (frame rate: $100 \mathrm{~Hz}$; Vicon System). Eight cameras were placed at a distance of 
1.5-2 $\mathrm{m}$ from the table on which the object was placed. Each participant was outfitted with 13 light-weight retroreflective hemispheric markers (4 $\mathrm{mm}$ in diameter) to create a hand model for kinematics analysis. Markers were placed on the dorsal aspect of the hand and the radial and the ulnar aspect of the wrist. Additional markers were placed at the tip, the metacarpo-phalangeal joint, the phalangeal-phalangeal joint of thumb, the index finger and the little finger, and on the trapezium bone of the thumb (Figure 1C).

After data collection, each trial was individually inspected for correct marker identification and then run through a low-pass Butterworth filter with a $6 \mathrm{~Hz}$ cutoff. We used a custom software (Matlab; MathWorks, Natick, MA, USA) to obtain the following kinematics parameters:

grip aperture, defined as the distance between the marker placed on thumb tip and that placed on the tip of the index finger (mm; see Figure 1C);

wrist velocity, defined as the module of the velocity of the wrist marker ( $\mathrm{mm} / \mathrm{sec}$; see rad in Figure 1C);

wrist height, defined as the $\mathrm{z}$-component of the wrist marker (mm).

All these variables were expressed with respect to the original frame of reference (i.e., the frame of reference of the motion capture system, termed as global frame of reference; Fglobal). In addition, the trajectory of the index and thumb finger were computed within a local frame of reference centerd on the hand (i.e., Flocal; see Ansuini et al., 2015; but also Carpinella et al., 2006, 2011 for a similar method). Flocal had its origin in the marker placed at the metacarpo-phalangeal joint of the index finger (see ind1 in Figure 1C).Vectors (ind1 - lit1) and (ind1 $\mathrm{rad}$ ) defined the metacarpal plane of the hand (refer to the shaded triangle in Figure 1C). In this frame of reference, the $\mathrm{x}$-axis had the direction of the vector (ind 1 - lit1) and pointed ulnarly, the $z$-axis was normal to the metacarpal plane, pointing dorsally, while the $y$-axis was calculated as the cross-product of $z$-and $x$-axes, pointing distally (see Figure 1C). Within this Flocal, we computed the following parameters:

$x-, y$-, and $z$-thumb, defined as $\mathrm{x}^{-}, \mathrm{y}^{-}$, and $\mathrm{z}$-coordinates for the marker placed on the tip of the thumb $(\mathrm{mm})$;

$x$-, $y$-, and $z$-index, defined as $\mathrm{x}$-, $\mathrm{y}^{-}$, and $\mathrm{z}$-coordinates for the marker placed on the tip of the index finger $(\mathrm{mm})$;

All these kinematics variables were expressed with respect to normalized (\%) rather than absolute (ms) movement durations. To this aim, we first computed time of reach onset (i.e., the first time point at which the wrist velocity crossed a $20 \mathrm{~mm} / \mathrm{sec}$ threshold and remained above it for longer than $100 \mathrm{~ms}$ ) and time of reach offset (i.e., the time at which the wrist velocity dropped below a $20 \mathrm{~mm} / \mathrm{s}$ threshold) to calculate movement duration (i.e., the time interval between reach onset and offset). In line with previous evidence (Goodale et al., 1994), analyses revealed that pantomimed movements were longer than real movements (average \pm SE: $944 \pm 55$ vs. $889 \pm 42$ ms; $p<0.05)$. Moreover, heavy compared to light target elicited longer movement durations (average \pm SE: $946 \pm 52$ vs. $887 \pm 44 \mathrm{~ms} ; p<0.05)$. Of interest, the effect of weight was identical in both real and pantomimed grasps (average \pm SE: $910 \pm 51$ vs. $978 \pm 59 \mathrm{~ms}$ and $864 \pm 39$ vs. $914 \pm 47 \mathrm{~ms}$ for light vs. heavy object in pantomimed and real movements, respectively; $p>0.05$ for 'Weight' by 'Condition' interaction). After normalizing the duration of each grasping movement, the data were resampled at intervals of 0.1 of the normalized movement time (resulting in decile increments of normalized reach duration).

To control for outliers, we $z$-transformed normalized data for each condition. Data points with $z$-scores less than -2.5 or greater than 2.5 were classified as statistical outliers and removed. Missing and outlier values $(<1.5 \%)$ were then replaced using Matlab File Exchange submission inpaint_nans ${ }^{1}$. This procedure interpolates and extrapolates based on sparse linear algebra and partial differential equations (PDE) discretization. A default method was used to solve approximations to PDEs using least squares approach in case of interpolation, while a linear behavior was applied for extrapolation (for a similar procedure see Ansuini et al., 2015).

\section{Statistical Analyses \\ Principal Component Analysis (PCA) of Kinematic Parameters}

To perform dimensionality reduction while retaining the maximum variation present in the original dataset and handling data collinearity (Næs and Mevik, 2001), we performed a principal component analysis (PCA) on the set of 90 variables, comprising the 9 spatial features (i.e., grip aperture, wrist velocity, wrist height, $x$-, $y$-, $z$-thumb, and $x$-, $y$-, $z$-index) across the 10 equally spaced temporal steps of the normalized reaching duration, for 1380 movements (60 over 1440 trials were discarded due to problems related to data recording). Principal Components (PCs) were extracted from a dataset where participants' data were pooled together rather than separated, thus applying the rule of thumb of higher observations per observed variable ratios in order to get more stable estimates (see Leonard, 2010). Furthermore, Bartlett's test of sphericity and Kaiser-Meyer-Olkin (KMO) measure of sampling adequacy were used to test for factorability (Bartlett, 1950; Kaiser, 1974). Both tests indicated that the sample was adequate for PCA (Bartlett's test: $\chi^{2}=410925,33$; d.f. $=4005 ; p<0.001$ and $\left.\mathrm{KMO}=0.828\right)$.

Mathematically, PCA consists of an orthogonal transformation which converts a set of $p$ variables $-\mathrm{x}_{1}, \mathrm{x}_{2}, \ldots . \mathrm{x}_{\mathrm{p}}$ of a matrix $\mathrm{X}$ (where variables are arranged in columns and observations are present in the rows) into $\mathrm{p}$ new uncorrelated PCs, $Z=\mathrm{z}_{1}$, $z_{2}, \ldots . z_{p}$. The PCs obtained are mutually uncorrelated in the sample and are arranged in decreasing order of their explained sample variances. The PC model, thus, transforms a data matrix $\mathrm{X}$ to a second matrix of $\mathrm{PC}$ scores, $\mathrm{Z}$ as $\mathrm{Z}=\mathrm{U}^{t} \mathrm{X}$, where the columns of $U=\mathrm{u}_{1}, \mathrm{u}_{2}, \ldots . \mathrm{u}_{\mathrm{p}}$ are the loading vectors, that is, the eigenvectors of the correlation matrix. In our case, the original data matrix $\mathrm{X}$ comprised of 90 kinematics variables in a time normalized domain (sampled at each $10 \%$ from $10 \%$ up

\footnotetext{
${ }^{1}$ http://www.mathworks.com/matlabcentral/fileexchange/4551-inpaint-nans
} 
to $100 \%$ of the movement duration) constituting the variables (columns) while all the 1380 trials from all the subjects were the observations (rows). To simplify data interpretation, we applied a varimax rotation to Principal Component axes to maximize the sum of the variances of the squared coefficients within each eigenvector (Kaiser, 1958). Kaiser's eigenvalue larger-thanone rule was applied to determine the number of significant components (Kaiser, 1960). The PCA led to the selection of the first 13 PCs as significant based on the selection of eigenvalues above 1. To obtain the lower dimension matrix based on the significant PCs, we generated component scores. Component scores are transformed variable values based on the constituent variables and their relative importance for a particular PC.

Mathematically, let $\mathrm{i}=1, \ldots, N$ index the rows (observations) and $j=1, \ldots, M$ index the columns (variables), then component score for a principal component $k$ for observation row $i,\left(Z_{\mathrm{k}, \mathrm{i}}\right)$ can be represented as:

$$
Z_{\mathrm{k}, \mathrm{i}}=u_{\mathrm{i} 1} * X_{\mathrm{i} 1}+u_{\mathrm{i} 2} * X_{\mathrm{i} 2}+\ldots \ldots u_{\mathrm{iM}} * X_{\mathrm{iM}}
$$

The component scores, thus, are a linear combination of the optimally weighted observed variables (Harman, 1976). This allowed us to obtain a lower dimension data set of component scores for all the PCs, with as many rows as original observations (i.e., 1380) and as many columns as the number of significant PCs (i.e., 13 PCs).

\section{Analysis of PCA Data using Linear Discriminant Analysis}

To determine the extent to which PCA data supported discrimination between the different movement categories, we submitted the output of the PCA to a LDA model (see Calder et al., 2001 for a similar procedure). Discriminant functions maximize the ratio of the between group variance $(\mathbf{B})$ to the within group variance $(\mathbf{W})$, in our instance, the groups being each of the four types of movements (i.e., real grasp_light object, real grasp_heavy object, pantomimed grasp_light object, pantomimed grasp_heavy object). The discriminant functions $y_{\mathrm{i}}$ are computed from the eigenvectors $l_{\mathrm{i}}$ of the ratio $\mathbf{W}^{-1} \mathbf{B}$ of the between group covariance matrix $(\mathbf{B})$ to the within group covariance matrix $(\mathbf{W})$ :

$$
y_{\mathrm{i}}=l_{\mathrm{i}} v
$$

where $v$ is the thirteen-dimensional vector of component scores. The relative size of each eigenvalue $\left(l_{i}\right)$ indicates the relative importance of each of the discriminant functions; rankordered according to the size of $l_{\mathrm{i}}$. The canonical discriminant function coefficients obtained from the eigenvectors express the contribution of each dependent variable to the different discriminant function (Field, 2013). Canonical $R^{2}$ (obtained by squaring canonical correlation for each discriminant function) was used as a measure of effect size (Field, 2013).

In $L D A$, the knowledge of the data class labels is used to find a low-dimensional representation that preserves the class differences, so that a classifier can be designed in the feature domain (Nenadic, 2007). For each of the four groups, we determined the location of the point representing the mean for all variables in the multivariate space defined by the variables in the model (i.e., centroids) and then computed the Mahalanobis distances (of the respective case) from each of the group centroids. Therefore, each case was classified as belonging to the group to which it was closest (i.e., where the Mahalanobis distance was smallest). A leave-one-out crossvalidation method was applied to evaluate the performance of the LDA model (Efron, 1982). In each round of this procedure, one case is held out from the dataset and assigned as a test for the classifier developed by using the remaining cases assigned as training set. This process is repeated until all the withheld cases in the dataset are validated and allows us to calculate the overall diagnostic accuracy of the LDA model. To investigate whether allocation distributions differed between expected (i.e., prior probabilities) and observed distributions (i.e., actual group membership), we applied Chi-squared test. Finally, to test whether classification scores significantly exceeded chance level, we randomly permuted the class labels and recomputed classification performance and a 95\% confidence interval (see Tritchler, 1984; Good, 2005; as implemented by a R package PredPsych $^{2}$ written in R; R Core Team, 2015). All analyses (except permutation testing) were performed using SPSS statistical software (version 21.0).

\section{RESULTS}

\section{Extracting Principal Components}

Thirteen PCs having eigenvalues above 1.00 accounted for $92 \%$ of the variance and all had communalities (i.e., amount of variance each component has in common with the set of all components; Field, 2013) greater than 0.70 (Dunteman, 1989; Stevens, 1996). It is a general rule to interpret variables with larger factor loadings as representative of the component (Hair et al., 1998). Here we followed this rule and considered factor loadings greater than 0.8 to load significantly on the component. Moreover, if the same variable loaded significantly onto more than one component, we considered the highest factor loading for interpreting the variable contribution on the corresponding component. A graphical representation (heat map) of all factor loadings (i.e., the factor loadings across all trials from all participants) for each variable is reported in Figure 2.

As can be seen, for the first seven PCs, high loadings $(>0.8)$ were found only for grip aperture and finger coordinates, suggesting that these PCs were related mainly to the distal aspect of the movement. In particular, the main contribution to PC1 originated from y-thumb (from $10 \%$ to $100 \%$ of normalized movement duration) and y-index (from 60\% to $100 \%$ of movement duration). Grip aperture between $20 \%$ and $60 \%$ of movement duration, $\mathrm{z}$-index between $30 \%$ and $60 \%$ of movement duration, and y-index between $20 \%$ and $50 \%$ of movement duration loaded significantly on PC2, while $\mathrm{x}$-thumb from $30 \%$ up to $100 \%$ of movement duration contributed significantly to PC3. For PC4, PC5, and PC6, higher factor loadings were found for $\mathrm{x}$-index between $30 \%$ and $100 \%$, z-thumb, and $\mathrm{z}$-index finger at the beginning of the movement (i.e., from $10 \%$ up

\footnotetext{
${ }^{2}$ https://github.com/ateshkoul/PredPsych
} 


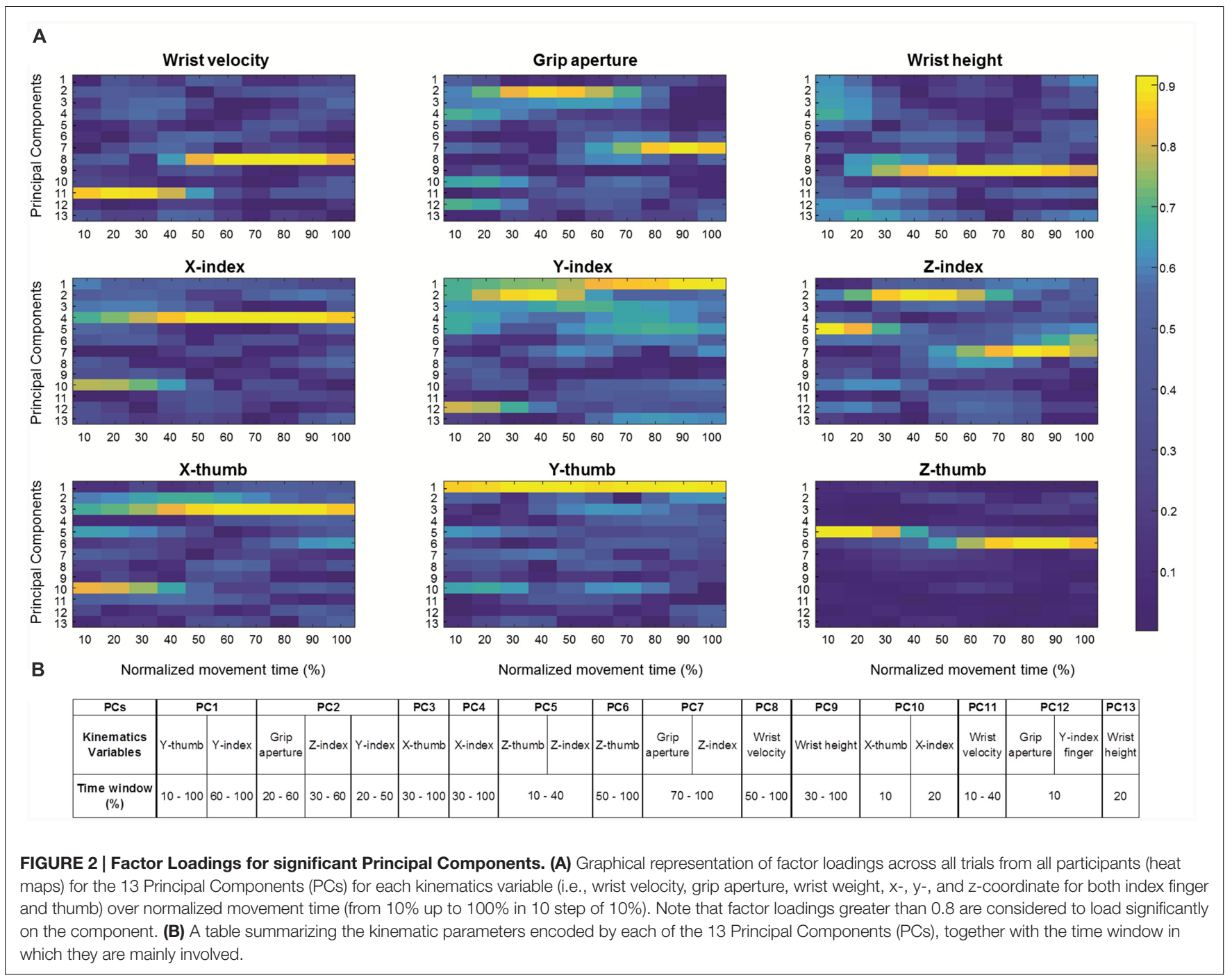

to $40 \%$ ), and for z-thumb from $50 \%$ up to $100 \%$ (Figure 2). Grip aperture between $70 \%$ and $100 \%$, and z-index within the same temporal interval loaded significantly on PC7. In contrast, kinematics parameters related to more proximal aspects of the movement were found to load significantly onto $P C 8, P C 9$, and PC11 (see Figure 2). In particular, wrist velocity from $10 \%$ up to $40 \%$ and from $50 \%$ up to the end of the movement contributed to $P C 11$ and $P C 8$, respectively, and wrist height from $30 \%$ up to the end of the movement loaded on PC9. Finally, an inspection of the factor loadings of $P C 10$ and $P C 12$, revealed large loadings of $\mathrm{x}$-thumb and $\mathrm{x}$-index at 10 and $20 \%$ of movement duration on $P C 10$, grip aperture and y-index finger at $10 \%$ on $P C 12$, and wrist height at $20 \%$ on PC13, suggesting that these components were associated mainly with the earliest phases of the movement.

\section{Identifying the Discriminant Functions for Different Movement Categories}

The LDA revealed that the first function accounted for $92.2 \%$ of the discriminating ability of the discriminating factors (eigenvalue equal to 2.085; canonical $R^{2}=0.68$ ), the second function for $7.1 \%$ (eigenvalue equal to 0.162 ; canonical $R^{2}=0.14$ ), and the third function for the remaining $0.7 \%$ (eigenvalue equal to 0.016; canonical $R^{2}=0.02$ ). As indicated by the chi-square tests performed on Wilks's lambda values ( $\lambda$ value $=0.275$; for $1^{\text {st }}$ st to $3^{\text {rd }}$ function, $2^{\text {nd }}$ nd to $3^{\text {rd }}$ function, and $3^{\text {rd }}$ function, respectively), the combination of the three functions provided a significant discriminative power $(p<0.05)$. A similar result was also found when considering the combination of the second and the third function as well as the contribution of the third function alone $(\lambda$ value $=0.847$; and 0.984 ; $\left.p_{\mathrm{s}}<0.05\right)$. Figure $3 \mathrm{~A}$ represents the canonical discriminant function scores for each observation, grouped according to the experimental condition to which that observation belonged. This graph, together with the values of the centroids, provides an intuitive visualization of how each function discriminates groups (Field, 2013). As apparent from this figure (please refer to $x$-axis), the first discriminant function mainly separated real and pantomimed grasping movements. The examination of the canonical discriminant function coefficients suggests that this 


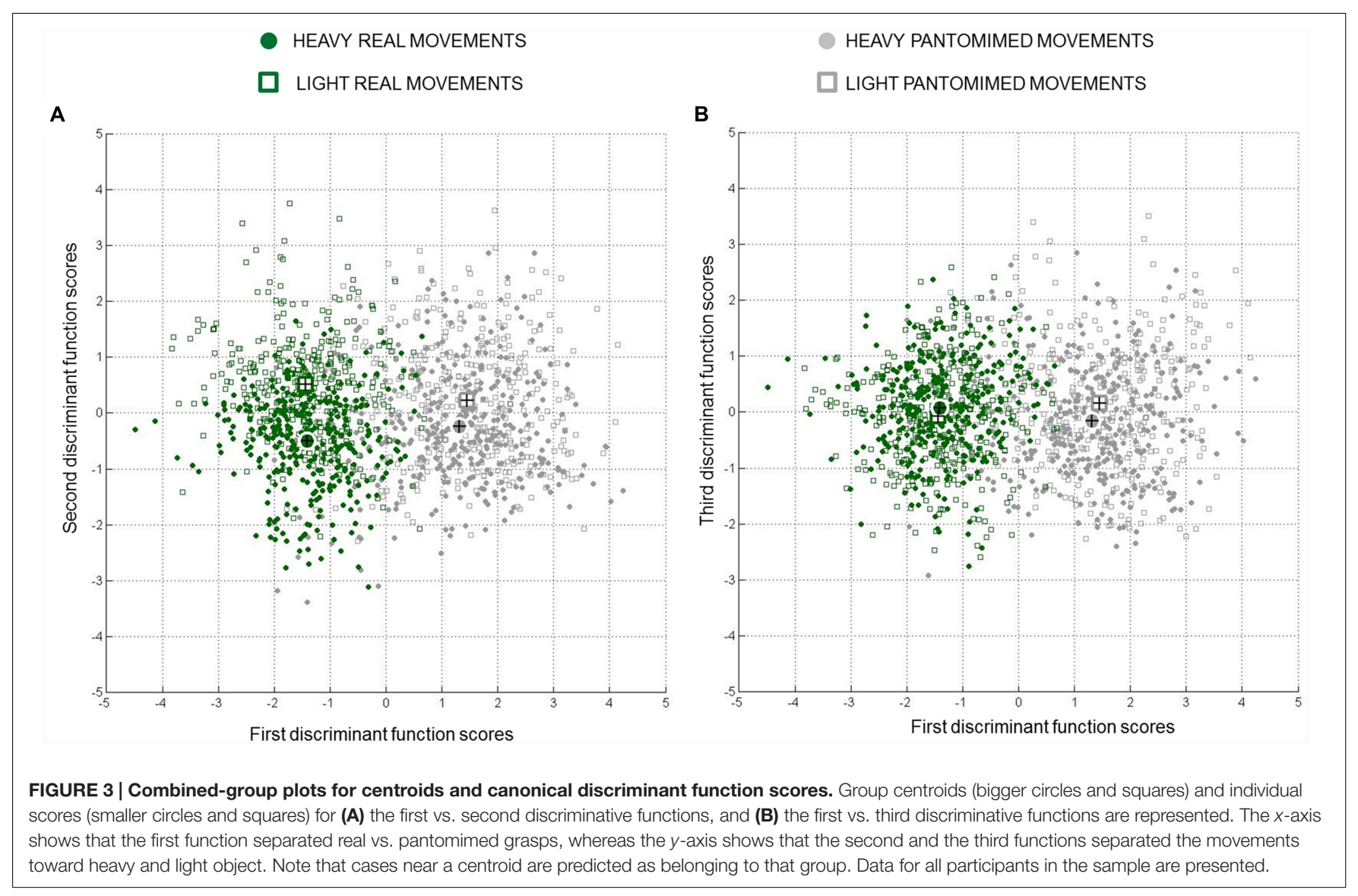

TABLE 1 | Canonical discriminant function coefficients for the three discriminant functions together with information related to the original kinematics variables that contributed the most to each principal component (PC).

\begin{tabular}{|c|c|c|c|c|}
\hline Original features contributing to principal component (PC) & PC number & $1^{\text {st }}$ function & $2^{\text {nd }}$ function & $3^{\text {rd }}$ function \\
\hline $\begin{array}{l}\text { Grip aperture (from } 20 \% \text { to } 60 \% \text { ), z-index (from } 30 \% \text { to } 60 \% \text { ), and y-index (from } 20 \% \text { to } \\
50 \% \text { ) }\end{array}$ & PC2 & 3,878 & 0,279 & $-0,763$ \\
\hline X-thumb (from $30 \%$ up to $100 \%$ ) & PC3 & $-1,067$ & 0,878 & $-0,478$ \\
\hline X-index (from $30 \%$ to $100 \%$ ) & PC4 & $-1,726$ & $-0,692$ & 1,177 \\
\hline Z-thumb and $z$-index finger (from $10 \%$ up to $40 \%$ ) & PC5 & 1,561 & $-0,988$ & $-0,785$ \\
\hline Wrist velocity (from 50\% up to $100 \%$ ) & PC8 & 4,062 & 1,557 & 0,544 \\
\hline Wrist height (from 30\% up to 100\%) & PC9 & 0,325 & 0,260 & 0,301 \\
\hline X-thumb (at 10\%) and $x$-index (at 20\%) & PC10 & 3,740 & 0,452 & $-0,714$ \\
\hline Wrist velocity (from $10 \%$ up to $40 \%$ ) & PC11 & 0,220 & 0,342 & 2,247 \\
\hline Grip aperture and y-index finger (at 10\%) & PC12 & 0,736 & 1,480 & $-1,746$ \\
\hline
\end{tabular}

Values in bold refer to dependent variables for which a significant canonical function correlation coefficient was found. Dependent variables with high canonical function correlations are usually interpreted as contributing the most to group separation (Bargman, 1970).

function was most dependent on $P C 7, P C 5$, and $P C 9$ (please refer to Table 1).

As evident from Figures $\mathbf{2 B}$ and $\mathbf{3}$, the $\mathrm{z}$-coordinate for index and thumb posture, wrist height, and grip aperture contributed the most to PC7, PC5, and PC9 so that these kinematics parameters were relatively more important than others for classifying the reality of the movement. It is worth noticing, however, that it is difficult to determine which kinematics behavior a PC is coding by simply inspecting the visual representation of its loadings. To complement this visual inspection of kinematics parameters across conditions over time, comparisons of interest were further explored by means 


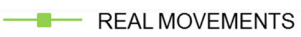

A

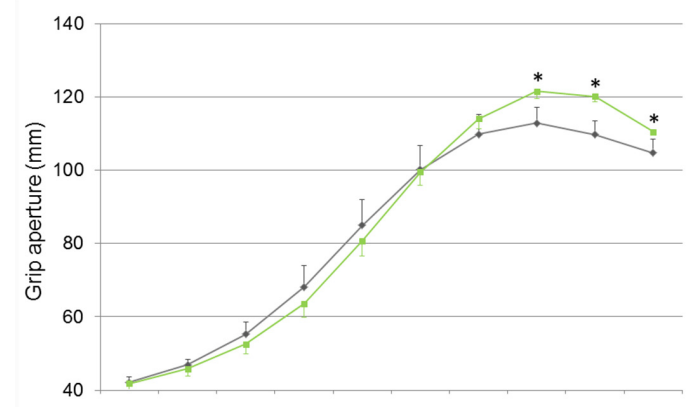

C

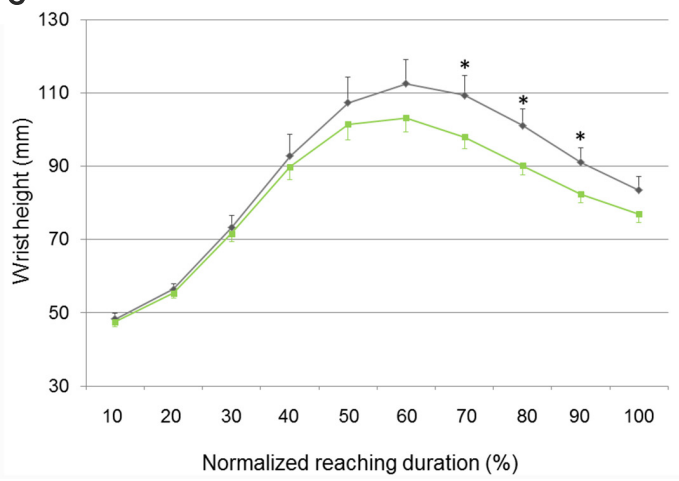

B

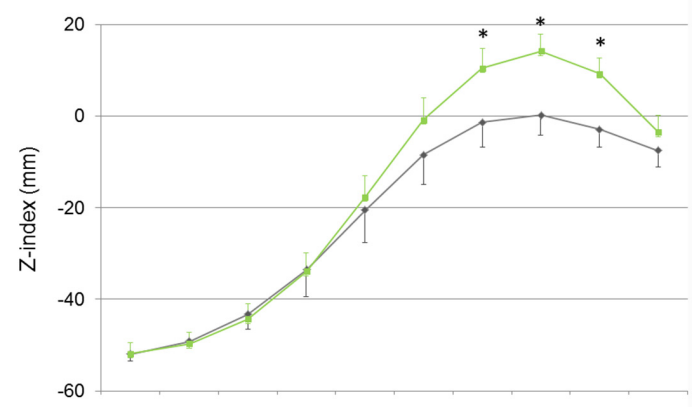

D

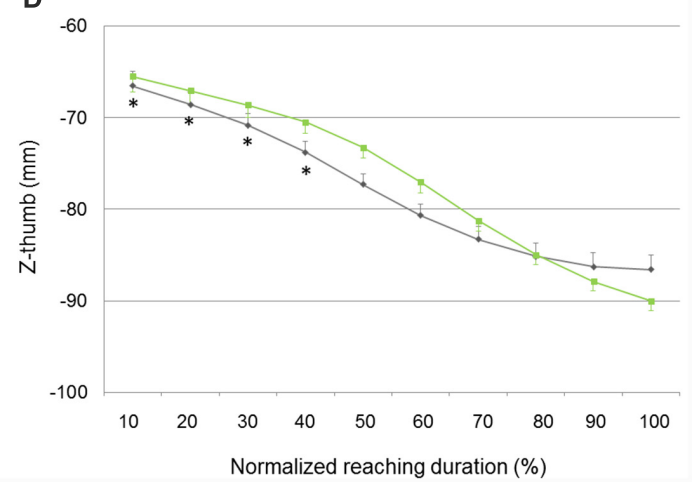

FIGURE 4 | Hand kinematics of real and pantomimed grasping movements. (A) Grip aperture, (B) z-index finger, (C) wrist height, and (D) z-thumb over time for real (green lines) and pantomimed (gray lines) grasping movements. Data are averaged across trials and participants. Bars represent standard error of the mean. Asterisks refer to statistical significance $(p<0.05)$.

of post hoc tests (with Bonferroni's correction). As shown in Figure 4A, for what concerns the first discriminant function, grip aperture was greater for real than for pantomimed grasping movement between $80 \%$ and $100 \%$ of normalized movement duration (PC7). Moreover, the index finger was less extended in the palmar direction (i.e., $\mathrm{z}$-index) in real than in the pantomimed movements from $70 \%$ up to $90 \%$ of normalized movement duration (PC7; Figure 4B). From $70 \%$ up to $90 \%$ of the reach-to-grasp movement, the wrist was higher when the movement was pantomimed rather than when it was real (PC9; Figure 4C). Finally, during the first part of the reaching movement (i.e., from $10 \%$ up to $40 \%$ of normalized movement duration), the thumb extended more dorsally (i.e., z-thumb) when the movement was real than when it was pantomimed (PC5; Figure 4D).

As illustrated in Figure 3A, the second discriminant function was more related to weight, supporting separation between real grasp movements performed toward heavy and light objects and, to a less extent, separation between pantomimed movements toward pretended heavy and light targets (please refer to $y$-axis in Figure $\mathbf{3 A}$ ). The PC6, PC3, PC10, PC11, and $P C 2$ correlated significantly with this second function (please refer to Table $\mathbf{1}$ for canonical discriminant function coefficients). Examining the kinematics parameters coded by these components (see Figure 2) revealed that, for both real and pantomimed movements, the thumb extended more dorsally (z-thumb; PC6) during the second phase of the movement when the target was heavy than when it was light (see Figures $\mathbf{5 A}$ and $\mathbf{6 A}$, respectively). Moreover, at about half of the reach-to-grasp movement, the grip aperture was smaller when the target was light than when it was heavy $(P C 2$; see Figures 5B and 6B). Other variables only expressed weight-related differences for real grasps. For example, wrist velocity between 10 and $40 \%$ of normalized movement time was greater for the heavy than for the light object for real but not for pantomimed movements (PC11; please refer to Figures 5C and 6C). Similarly, for real grasps but not for pantomimed grasps, the index finger was less extended in palmar direction and pointed more distally ( $\mathrm{z}$-index and $\mathrm{y}$-index; $P C 2$ ), and the thumb pointed more radially (x-thumb; PC3), for the heavy object than for the light object Figures 5D-F and $6 \mathrm{D}-\mathrm{F}$, respectively). Finally, since the $\mathrm{x}$-coordinate of index finger (PC10) did not express significant weight-related differences in either pantomimed or real movements $\left(p_{\mathrm{s}}>0.05\right)$, no clear interpretation for corresponding component was possible.

For what concerns the third discriminative function, the inspection of Figure 3B suggests that this function separated 

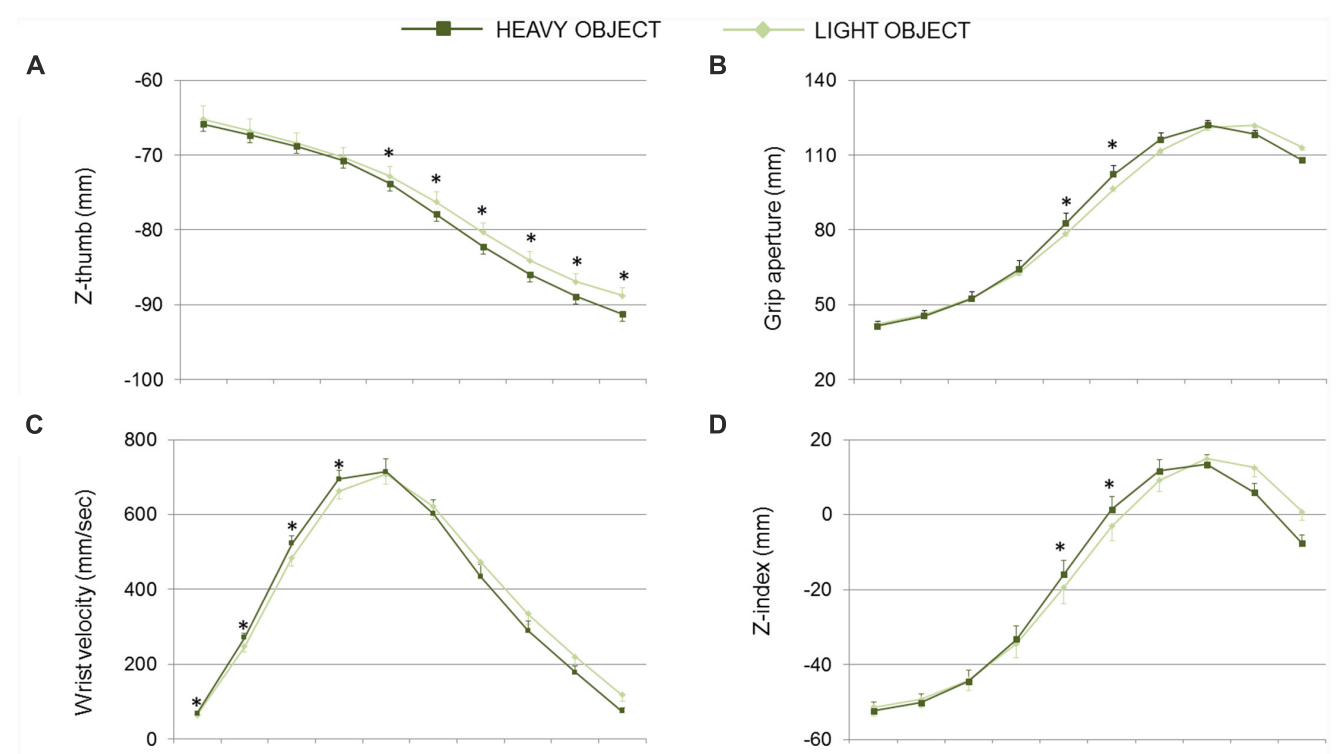

D

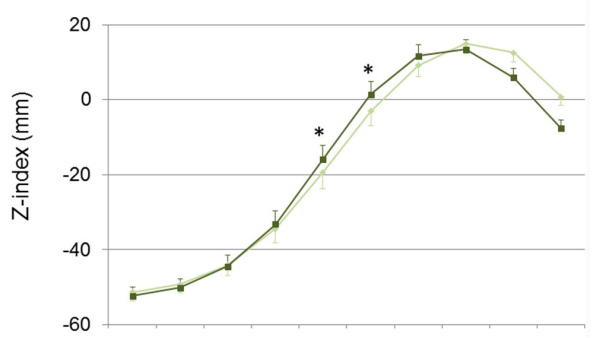

$\mathrm{E}$

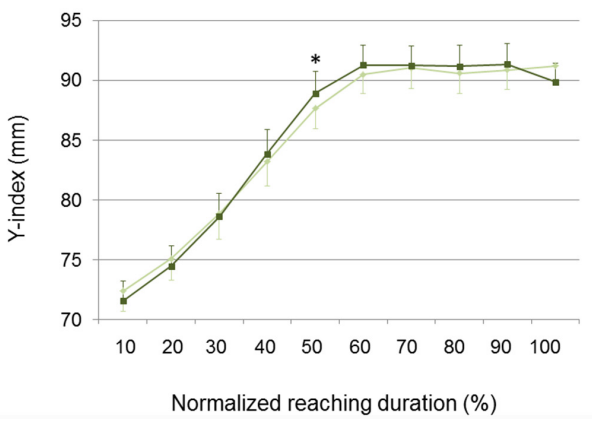

$\mathbf{F}$

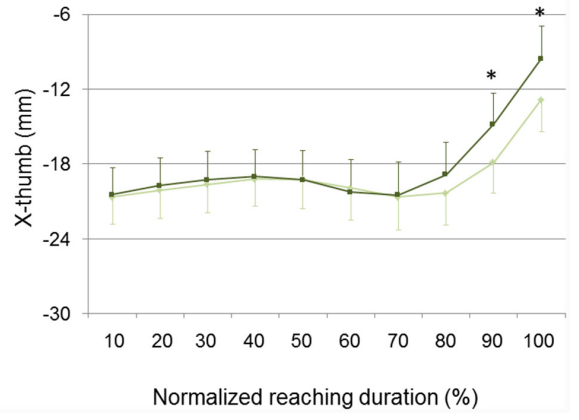

FIGURE 5 | Hand kinematics of real grasping movements toward light and heavy objects. (A) Z-thumb, (B) grip aperture, (C) wrist velocity, (D) z-index finger, (E) y-index finger, and (F) x-thumb over time for movements toward heavy and light object (dark and light green lines, respectively). Data are averaged across trials and participants. Bars represent standard error of the mean. Asterisks refer to statistical significance $(p<0.05)$.

cases based on the weight of the target object. Interestingly, the inspection of centroids suggests that the separation along this function was more pronounced for pantomimed grasps than for real grasps ('real grasp' $=-0.070$ and -0.078 for light object and heavy object, respectively, and 'pantomimed grasp' $=0.154$ and -0.167 for light object and heavy object, respectively; see Figure 3B). This function, however, accounted for only a marginal portion of $0.7 \%$ of the total variance. Caution is therefore needed when interpreting the kinematics parameters coded by the correlated PCs (PC12, PC4, PC13, PC1, and PC8; please refer to Table $\mathbf{1}$ for canonical discriminant function coefficients).

\section{Classification of Object Weight}

Table 2A reports the confusion matrix for the LDA model from the set of PCA data. As can be seen, in each of the four categories, reach-to-grasp movements were classified with above chance accuracy $\left(\chi^{2}{ }_{(9)}=1.207,8 ; p<0.05\right.$ with an $a$ priori probability equal to $25 \%$ ). In particular, for real grasps, movements toward light and heavy objects were correctly classified on 68 and $67 \%$ of cases, respectively, whereas for pantomimed grasps, correct classification of movements toward pretended light and heavy objects occurred in 53 and $49 \%$ of cases, respectively.

However, since the probability of light vs. heavy classification interacts with the probability of real vs. pantomimed classification, these results might overstate the effect of object weight. In order to adopt a more conservative approach to quantify the impact of weight, we therefore proceeded to perform two separate LDAs for real and pantomimed grasps. Table 2B reports the confusion matrices for these analyses. Although the overall proportion of correct classification suffered, classification of object weight was still significantly above chance level for both real and pantomimed grasps. To further support this conclusion, we also performed permutation tests to assess whether correct classification scores were significantly above chance level. By randomly permuting the class labels and recomputing classification performance, we confirmed that the classification scores were indeed significant $\left[\left(p_{\mathrm{s}}<0.001\right), 95 \%\right.$ Confidence Intervals: All four movements (0\%, 27\%); light/heavy for real movements $(0 \%, 54 \%)$; light/heavy for pantomimed movements (0\%, 53\%) (Tritchler, 1984; Good, 2005)]. 


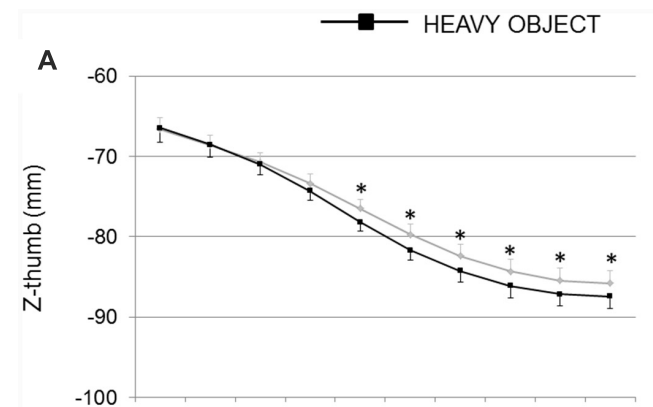

C

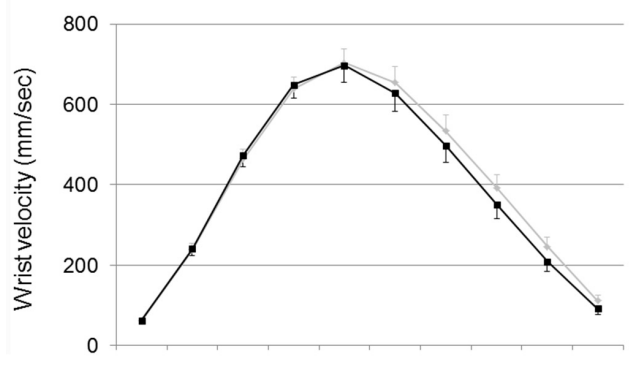

E

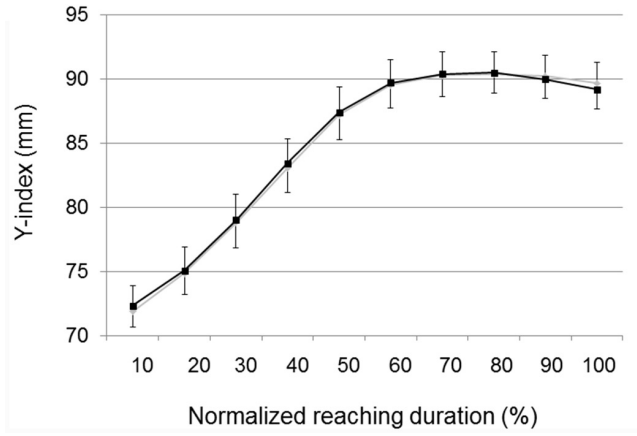

B

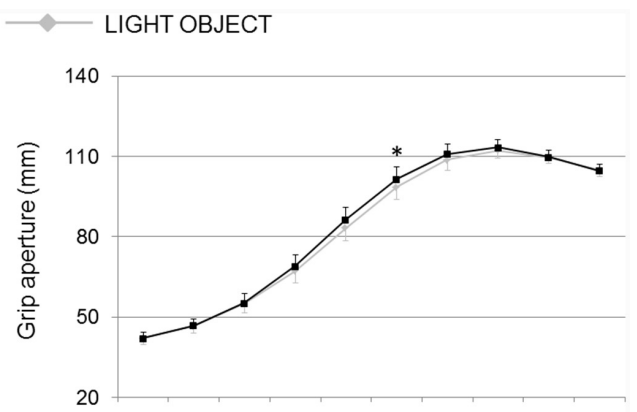

D

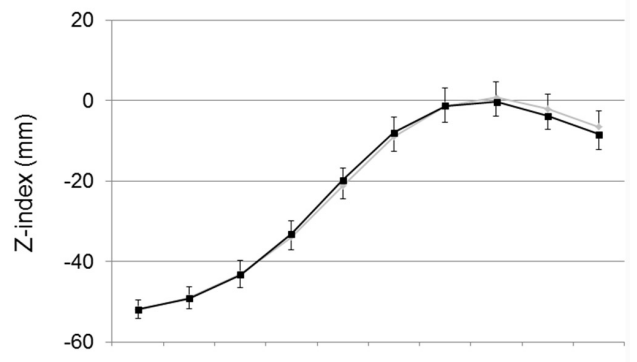

$\mathbf{F}$

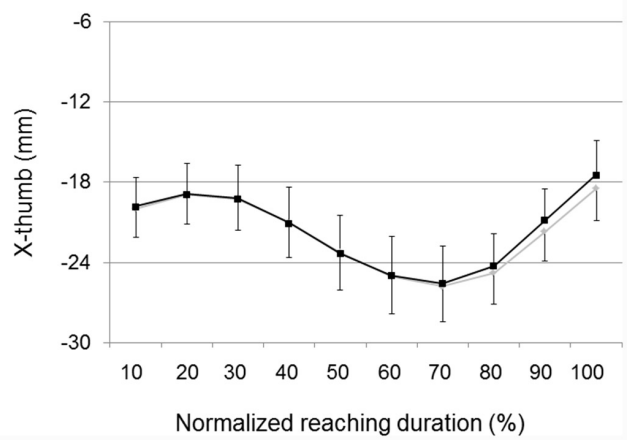

FIGURE 6 | Hand kinematics of pantomimed grasping movements toward pretended light and heavy objects. (A) Z-thumb, (B) grip aperture, (C) wrist velocity, (D) z-index finger, (E) y-index finger, and (F) x-thumb over time for movements toward heavy and light object (black and light gray lines, respectively). Data are averaged across trials and participants. Bars represent standard error of the mean. Asterisks refer to statistical significance $(p<0.05)$.

\section{DISCUSSION}

Previous research on the relationship between reach-to-grasp movement and the properties of the to-be-grasped object indicates that object weight influences pre-contact kinematics in preparation for a stable final grip placement on the object (Weir et al., 1991; Brouwer et al., 2006; Eastough and Edwards, 2007). Heavy compared to light objects cause increased peak grip aperture, a final finger and thumb placement on the object that more closely passes through the center of mass of the object, and a reduced peak lift velocity (Eastough and Edwards, 2007). Our results confirm and extend these findings by showing that early on in the movement, hand kinematics of real grasps is already scaled to the weight of the to-be-grasped object. As shown in Figure 5, the thumb extended more dorsally when the target was heavy than when it was light. Moreover, early on in the reach, grip aperture was larger and wrist velocity was higher for heavy than for light objects. As shown by LDA, prior-to-contact kinematics conveyed indeed enough information to discriminate between real grasp movements aimed at heavy and light objects.

Remarkably, when we examined pantomimed grasp, we found that classification accuracy for heavy vs. light object was lower, but still significantly above the chance level. As for real grasp movements, in the last part of the movement, the thumb extended more dorsally (z-thumb) when the pretended target was heavy than when it was light. Other kinematics parameters sensitive to object weight for real grasp movements, however, showed no similar weight-attunement for pantomimed grasp. For example, whereas the thumb pointed more ulnarly and the index finger pointed more radially for real grasps aimed at a heavy object, no similar modulation was observed for pantomimed movements. In the following, we examine in some details three factors that may have contributed to the differential modulatory effect of weight on real and pantomimed grasps.

A first factor to consider is the removal of the physical object per se. During real grasps, the mechanical properties of the 
TABLE 2 | Confusion matrix from linear discriminant analyses for (A) the four movement type by object weight categories and (B) the two object weight categories for real and pantomimed movements separately, applied to the sets of PCA data (i.e., 13 Principal Components).

\begin{tabular}{|c|c|c|c|c|c|c|}
\hline & & Light & Heavy & Light & Heavy & Total \\
\hline \multirow[t]{2}{*}{ Real } & Light & $68 \%(231)$ & $27 \%(91)$ & $3 \%(9)$ & $2 \%(6)$ & $100 \%(337)$ \\
\hline & Heavy & 29\% (99) & $67 \%(231)$ & $2 \%(6)$ & $2 \%(7)$ & $100 \%(343)$ \\
\hline \multicolumn{7}{|l|}{ (B) } \\
\hline \multirow[t]{2}{*}{ Real } & Light & $74 \%$ (249) & 26\% (88) & & & $100 \%(337)$ \\
\hline & Heavy & $26 \%(90)$ & $74 \%$ (253) & & & $100 \%(343)$ \\
\hline \multirow[t]{2}{*}{ Pantomimed } & Light & & & $60 \%(212)$ & $40 \%(142)$ & $100 \%$ (354) \\
\hline & Heavy & & & $42 \%(145)$ & $58 \%(201)$ & $100 \%$ (346) \\
\hline
\end{tabular}

Note that the grey diagonal highlights cross-validated grouped cases correctly classified. Actual number of observations is shown in parentheses.

object (such as its weight) are critical for motor control. During pantomime, in contrast, the participant's hand does not come into contact with a material object, but only with 'thin air.' Without actual interaction between the hand and the target, there are no obvious consequences for an inaccurate grasping (e.g., the slippage or the roll of the object), permitting one to neglect motor programs that adapt the hand to the material object. This could explain the reduced attunement to weight for pantomimed in comparison to real grasps.

A second factor - causally related to first - refers to the specific role of haptic-based information in sensorimotor transformations supporting prehensile actions. Interestingly, whereas haptic feedback is per se not sufficient to evoke motor programs for correct tool use (Goldenberg et al., 2004), there is evidence that removing haptic feedback shifts the response mode from a real one toward a pantomimed one. Even when the movements are directed toward a visible virtual target (viewed in a mirror), removing haptic feedback has been shown to influence grasp kinematics such that grasps without haptic feedback are statistically indistinguishable from pantomimed grasps (Whitwell et al., 2015). The fundamental role of haptic feedback in hand tuning is further supported by evidence from DF, a patient who suffered from visual form agnosia (Schenk, 2012). By using a mirror-apparatus to dissociate the image of an object from its physical presence, it was shown that, without haptic feedback, DF's grasping performance was not better than her (poor) performance in the manual estimation task (i.e., matching the distance between the thumb and the index finger to the size of the object). Crucially, when intermittent haptic feedback was provided, DF's performance improved (Schenk, 2012). On this account, the patterning of pantomimed grasp would thus reflect the absence of haptic-based object information.

Removal of the physical object or, more specifically, absence of tactile feedback, however, may be not enough to explain the differential features of pantomimed grasps. Pantomime neglects features of the object that are important for manipulation but have little value for discriminating the object, whereas it specifies features that in actual use are determined by the manipulated object.

A third factor to consider relates thus to the deliberate process of demonstrating the properties of the pretended target (Goldenberg et al., 2003). We speculate that the kinematics of pantomimed actions may convey information about the symbolic motor representation of the pretended weight (Goldenberg et al., 2003; see also, Hermsdörfer et al., 2005, 2006; Laimgruber et al., 2005). However, we wish to emphasize that these considerations are of a very speculative nature because participants in our study were not explicitly instructed to communicate the weight of the object. An interesting prediction for future studies is that explicitly instructing participants to communicate object weight to another person should increase weight discriminability for pantomimed grasp.

Related to this, it will be interesting to investigate to what extent observers watching a pantomimed grasp are able to infer the properties of the pretended object. Some behavioral studies already indicate that the weight of an object (e.g., a box) can be inferred quite accurately when observing another person lifting it (Runeson and Frykholm, 1983; Bingham, 1987; Hamilton et al., 2004). Moreover, there is evidence that muscle-specific M1 excitability modulates to the force requirements of observed object lifting (i.e., M1 excitability is considerably higher when observing heavy object lifting compared to light object lifting) and that this modulation is sensitive to the kinematics conveyed by the observed action (Alaerts et al., 2010a,b). To our knowledge, however, no previous study has investigated whether observers are able to read out the weight of a to-be-grasped object from pre-contact kinematics. Moreover, there is no information in the literature regarding observers' ability to infer object weight from pantomimed grasps. The classification results in our study lend some plausibility to this hypothesis by showing that precontact kinematics provide a firm informational basis for weight discrimination for real grasps and - albeit to a lesser extent - for pantomimed grasp. In future research, we plan to test whether and to what extent observers are able to make use of this information to discriminate weight and other non-spatial object 
properties (such as object fragility). Future research should also focus on the extent to which real and pantomimed grasps convey a categorical representation of weight information (i.e., an object is either heavy or light) versus a continuous representation of weight (i.e., changes in activity patterns that directly correspond to changes in object weight) and on the exact time course of weight specification (i.e., how weight information is specified at specific time intervals).

Finally, it will be important to consider these results from the perspective of the neural mechanism involved in extracting object weight when pantomiming a reach-to-grasp movement. Consistent with the proposed division of labor in the visual pathways of the primate cerebral cortex, between a dorsal pathway specialized for action control and a ventral stream dedicated to the perception of the visual world (Milner and Goodale, 1995), processing object features critical for motor control, such as object weight, has been traditionally thought to be in the purview of the dorsal pathway. Recent functional neuroimaging (fMRI) evidence, however, suggests that, in addition to traditional motor-related areas, the lateral occipital cortex (LOC) in the ventral visual stream represents object weight when preparing to lift an object (Gallivan et al., 2014). Expanding upon this result, it is tempting to speculate that the LOC representation of object weight may inform and support weight-related pantomime. Functional neuroimaging studies and patient studies may help to clarify the differential

\section{REFERENCES}

Alaerts, K., Senot, P., Swinnen, S. P., Craighero, L., Wenderoth, N., and Fadiga, L. (2010a). Force requirements of observed object lifting are encoded by the observer's motor system: a TMS study. Eur. J. Neurosci. 31, 1144-1153. doi: 10.1111/j.1460-9568.2010.07124.x

Alaerts, K., Swinnen, S. P., and Wenderoth, N. (2010b). Observing how others lift light or heavy objects: which visual cues mediate the encoding of muscular force in the primary motor cortex? Neuropsychologia 48, 2082-2090. doi: 10.1016/j.neuropsychologia.2010.03.029

Ansuini, C., Cavallo, A., Koul, A., Jacono, M., Yang, Y., and Becchio, C. (2015). Predicting object size from hand kinematics: a temporal perspective. PLoS ONE 10:e0120432. doi: 10.1371/journal.pone.0120432

Bargman, R. E. (1970). "Interpretation and use of a generalized discriminant function," in Essays in Probability and Statistics, eds R. C. Bose, I. M. Chakravarti, P. C. Mahalanobis, C. R. Rao, and K. J. C. Smith (Chapel Hill, NC: University of North Carolina), 35-60.

Bartlett, M. S. (1950). Tests of significance in factor analysis. Br. J. Stat. Psychol. 3, 77-85. doi: 10.1111/j.2044-8317.1950.tb00285.x

Bingham, G. P. (1987). Kinematic form and scaling: further investigations on the visual perception of lifted weight. J. Exp. Psychol. Hum. Percept Perform. 13, 155-177. doi: 10.1037/0096-1523.13.2.155

Brouwer, A. M., Georgiou, I., Glover, S., and Castiello, U. (2006). Adjusting reach to lift movements to sudden visible changes in target's weight. Exp. Brain Res. 173, 629-636. doi: 10.1007/s00221-006-0406-x

Calder, A. J., Burton, A. M., Miller, P., Young, A. W., and Akamatsu, S. (2001). A principal component analysis of facial expressions. Vision Res. 41, 1179-1208. doi: 10.1016/S0042-6989(01)00002-5

Carpinella, I., Jonsdottir, J., and Ferrarin, M. (2011). Multi-finger coordination in healthy subjects and stroke patients: a mathematical modelling approach. J. Neuroeng. Rehabil. 8:19. doi: 10.1186/1743-0003-8-19

Carpinella, I., Mazzoleni, P., Rabuffetti, M., Thorsen, R., and Ferrarin, M. (2006). Experimental protocol for the kinematic analysis of the hand: definition and repeatability. Gait Posture 23, 445-454. doi: 10.1016/j.gaitpost.2005.05.001 contribution of the ventral and the dorsal pathways to object weight processing in preparation of real and pantomimed grasps.

\section{AUTHOR CONTRIBUTIONS}

CA: designed the experiment, analyzed the data and wrote the article. AC: designed and performed the experiment. CC: analyzed the data. AK: performed the experiment and analyzed the data. DQ: performed the experiment. CB: designed the experiment, supervised the work and wrote the article.

\section{FUNDING}

This work received funding from the European Research Council under the European Union's Seventh Framework Programme (FP7/2007-2013)/ERC grant agreement n. 312919.

\section{ACKNOWLEDGMENTS}

Authors would like to thank Marco Jacono for his help with kinematics measuring and Luca Pascolini for his help with data pre-processing.

Cavina-Pratesi, C., Kuhn, G., Ietswaart, M., and Milner, A. D. (2011). The magic grasp: motor expertise in deception. PLoS ONE 6:e16568. doi: 10.1371/journal.pone.0016568

Douglas, P. H., and Moscovice, L. R. (2015). Pointing and pantomime in wild apes? Female bonobos use referential and iconic gestures to request genito-genital rubbing. Sci. Rep. 5:13999. doi: 10.1038/srep13999

Dunteman, G. H. (1989). Principal Components Analysis (No. 69). Thousand Oaks, CA: Sage.

Eastough, D., and Edwards, M. G. (2007). Movement kinematics in prehension are affected by grasping objects of different mass. Exp. Brain Res. 176, 193-198. doi: 10.1007/s00221-006-0749-3

Efron, B. (1982). The Jackknife, the Bootstrap and other Resampling Plans, Vol. 38. Philadelphia, PA: Society for industrial and applied mathematics.

Field, A. (2013). Discovering Statistics Using IBM SPSS Statistics. London: Sage.

Gallivan, J. P., Cant, J. S., Goodale, M. A., and Flanagan, J. R. (2014). Representation of object weight in human ventral visual cortex. Curr. Biol. 24, 1866-1873. doi: 10.1016/j.cub.2014.06.046

Goldenberg, G. (2013). Apraxia. Wiley Interdiscip. Rev. Cogn. Sci. 4, 453-462. doi: $10.1002 /$ wcs. 1241

Goldenberg, G., Hartmann, K., and Schlott, I. (2003). Defective pantomime of object use in left brain damage: apraxia or asymbolia? Neuropsychologia 41, 1565-1573. doi: 10.1016/S0028-3932(03)00120-9

Goldenberg, G., Hentze, S., and Hermsdörfer, J. (2004). The effect of tactile feedback on pantomime of tool use in apraxia. Neurology 63, 1863-1867. doi: 10.1212/01.WNL.0000144283.38174.07

Goldenberg, G., Hermsdörfer, J., Glindemann, R., Rorden, C., and Karnath, H. O. (2007). Pantomime of tool use depends on integrity of left inferior frontal cortex. Cereb. Cortex 17, 2769-2776. doi: 10.1093/cercor/ bhm004

Good, P. (2005). Permutation, Parametric and Bootstrap Tests of Hypotheses. New York, NY: Springer-Verlag. doi: 10.1007/b138696

Goodale, M. A., Jakobson, L. S., and Keillor, J. M. (1994). Differences in the visual control of pantomimed and natural grasping movements. Neuropsychologia 32, 1159-1178. doi: 10.1016/0028-3932(94)90100-7 
Hair, J. F., Tatham, R. L., Anderson, R. E., and Black, W. (1998). Multivariate Data Analysis, 5th Edn. London: Prentice-Hall.

Hamilton, A. D. C., Wolpert, D. M., and Frith, U. (2004). Your own action influences how you perceive another person's action. Curr. Biol. 14, 493-498. doi: $10.1016 /$ j.cub.2004.03.007

Harman, H. H. (1976). Modern Factor Analysis, 3rd Edn. Chicago, IL: University of Chicago Press.

Hermsdörfer, J., Hentze, S., and Goldenberg, G. (2006). Spatial and kinematic features of apraxic movement depend on the mode of execution. Neuropsychologia 44, 1642-1652. doi: 10.1016/j.neuropsychologia.2006. 03.023

Hermsdörfer, J., Nowak, D. A., Lee, A., Rost, K., Timmann, D., Mühlau, M., et al. (2005). The representation of predictive force control and internal forward models: evidence from lesion studies and brain imaging. Cogn. Process. 6, 48-58. doi: 10.1007/s10339-004-0042-y

Jeannerod, M. (1988). The Neural and Behavioural Organization of Goal-Directed Movements. Oxford: Clarendon Press/Oxford University Press.

Kaiser, H. F. (1958). The varimax criterion for analytic rotation in factor analysis. Psychometrika 23, 187-200. doi: 10.1007/BF02289233

Kaiser, H. F. (1960). The application of electronic computers to factor analysis. Educ. Psychol. Meas. 20, 141-151. doi: 10.1177/001316446002000116

Kaiser, H. F. (1974). An index of factorial simplicity. Psychometrika 39, 31-36. doi: 10.1007/BF02291575

Laimgruber, K., Goldenberg, G., and Hermsdörfer, J. (2005). Manual and hemispheric asymmetries in the execution of actual and pantomimed prehension. Neuropsychologia 43, 682-692. doi: 10.1016/j.neuropsychologia. 2004.09.004

Leonard, K. A. (2010). Sample size and subject to item ratio in principal components analysis and exploratory factor analysis. J. Biom. Biostat 1:106. doi: 10.4172/2155-6180.1000106

Milner, A. D., and Goodale, M. A. (1995). The Visual Brain in Action, Vol. 27. Oxford: Oxford University Press.

Nenadic, Z. (2007). Information discriminant analysis: feature extraction with an information-theoretic objective. IEEE Tran. Pattern Anal. Mach. Intell. 29, 1394-1407. doi: 10.1109/TPAMI.2007.1156

Næs, T., and Mevik, B. H. (2001). Understanding the collinearity problem in regression and discriminant analysis. J. Chemometr. 15, 413-426. doi: $10.1002 / \mathrm{cem} .676$

R Core Team (2015). R: A Language and Environment for Statistical Computing. Vienna: R Foundation for Statistical Computing.
Runeson, S., and Frykholm, G. (1983). Kinematic specification of dynamics as an informational basis for person-and-action perception: expectation, gender recognition, and deceptive intention. J. Exp. Psychol. Gen. 112:585. doi: 10.1037/0096-3445.112.4.585

Schenk, T. (2012). No dissociation between perception and action in patient DF when haptic feedback is withdrawn. J. Neurosci. 32, 2013-2017. doi: 10.1523/JNEUROSCI.3413-11.2012

Smeets, J. B., and Brenner, E. (1999). A new view on grasping. Motor Control 3, 237-271. doi: $10.1123 /$ mcj.3.3.237

Stevens, J. P. (1996). Applied Multivariate Statistics for the Social Sciences, 4th Edn. Mahwah, NJ: Lawrence Erlbaum Associates.

Tritchler, D. (1984). On Inverting Permutation Tests. J. Am. Stat. Assoc. 79, 200-207. doi: 10.2307/2288357

Weir, P. L., MacKenzie, C. L., Marteniuk, R. G., Cargoe, S. L., and Frazer, M. B. (1991). The effects of object weight on the kinematics of prehension. J. Motor Behav. 23, 192-204. doi: 10.1080/00222895.1991. 10118362

Westwood, D. A., Heath, M., and Roy, E. A. (2000). The effect of a pictorial illusion on closed-loop and open-loop prehension. Exp. Brain Res. 134, 456-463. doi: $10.1007 / \mathrm{s} 002210000489$

Whitwell, R. L., Ganel, T., Byrne, C., and Goodale, M. A. (2015). Real-time vision, tactile cues, and visual form agnosia in pantomimed grasping: removing haptic feedback induces a switch from natural to pantomimelike grasps. Front. Hum. Neurosci. 9:216. doi: 10.3389/fnhum.2015. 00216

World Medical Association General Assembly (2008). Declaration of Helsinki. Ethical principles for medical research involving human subjects. World Med. J. 54, 122-125.

Conflict of Interest Statement: The authors declare that the research was conducted in the absence of any commercial or financial relationships that could be construed as a potential conflict of interest.

Copyright (C) 2016 Ansuini, Cavallo, Campus, Quarona, Koul and Becchio. This is an open-access article distributed under the terms of the Creative Commons Attribution License (CC BY). The use, distribution or reproduction in other forums is permitted, provided the original author(s) or licensor are credited and that the original publication in this journal is cited, in accordance with accepted academic practice. No use, distribution or reproduction is permitted which does not comply with these terms. 